\title{
Simulation on Vehicular Ad-hoc Networks Using Proposed BBICR Technique
}

\author{
Shuchi Smita ${ }^{1}$, Ashutosh Kumar ${ }^{2}$, K Suresh ${ }^{3}$ \\ M Tech Student, School of Computer Science and Engineering, Galgotias University, Noida, India ${ }^{1}$ \\ Assistant Professor, Department of Civil Engineering, \\ Datta Meghe Institute of Engineering Technology \& Research, Wardha, India ${ }^{2}$ \\ Associate Professor, School of Computer Science and Engineering, Galgotias University, Noida, India ${ }^{3}$
}

\begin{abstract}
In vehicular ad hoc network specification, the border node can be selected based on one-hop neighbor data using a minimum neighbor based distance concept, such approach for the various protocol where nodes locate nearest neighbor positions lists, and follow a distributed network-based procedure, thus determine which vehicle/nodes share the least number of common neighbors. In this paper proposed, the node which satisfy present state are typically outermost from next forwarding node of border side of intercommunication system model with border based routing make hybridization, that minimizing end to end delay and improving average throughput with our hybrid protocol that is BBICR, using MATLAB 2014Ra version.
\end{abstract}

Keywords: VANET, BBR, ICS, Packet Forwarding, Neighbor Node etc

\section{INTRODUCTION}

VANET stand for the Vehicular Ad-hoc Network. A VANET is a new type of Mobile Ad-hoc Network (MANET) that is a self-framing network and exceptionally adaptable, which has a few characteristics like dynamic topologies, less transmission capacity and energy consumption and it can work without the need of any concentrated control. In this manner, VANET is wireless communication among vehicle to vehicle for different area vehicle to a roadside framework in light of Wireless Local Area Network (WLAN) innovation. Every hub in an ad hoc network goes about as both an information terminal and a switch $[1,2]$. The hubs in the network at that point utilize the wireless medium to speak with different hubs in their radio range. The advantage of utilizing ad hoc networks is that it is conceivable to convey these networks in areas where it isn't doable to introduce the required framework. Another significance of ad hoc networks is that it could be immediately conveyed with no administrator association. The administration of a substantial scale vehicular network would be a troublesome assignment. These reasons add to the ad hoc networks being connected to vehicular environments. Fig.1 [3] shows a vehicular ad-hoc network with all its working. The moving vehicles are communicating with each other and as well as to the Road Side Units (RSU). Different roadside units are also communicating with each other and as well as with the infrastructure.

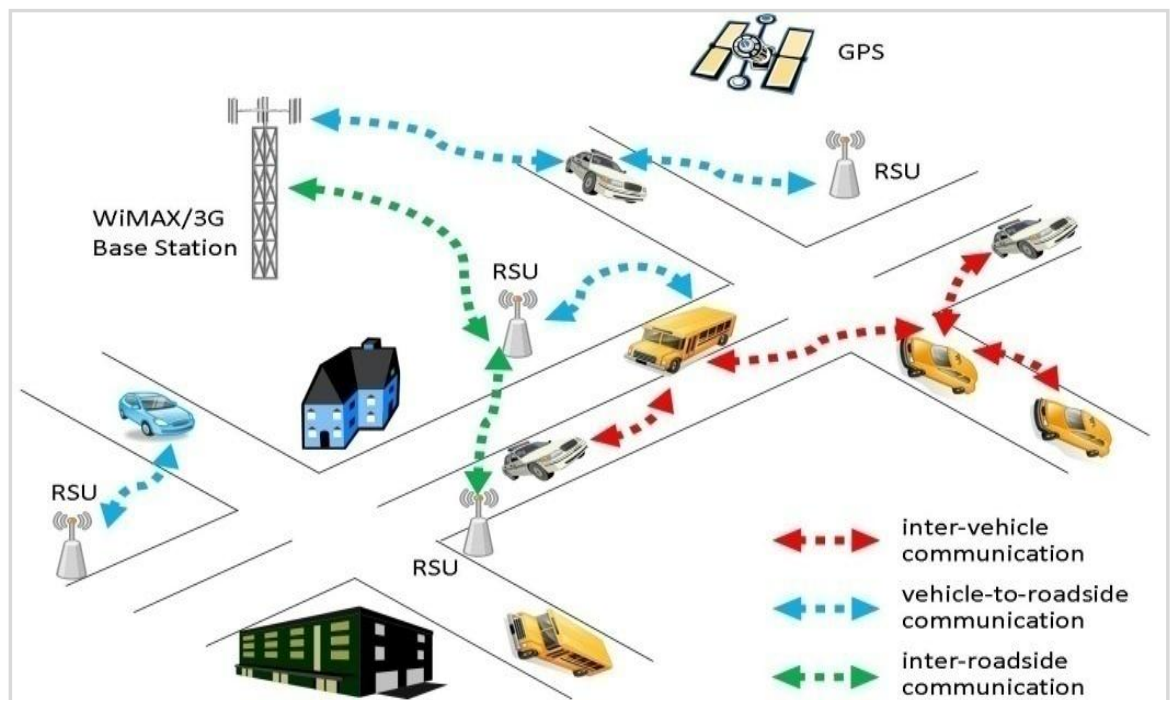

Fig. 1 Vehicular Ad Hoc Network 


\title{
International Advanced Research Journal in Science, Engineering and Technology
}

\author{
Vol. 5, Issue 9, September 2018
}

A.

Components of vanets

There are three components of VANET:

(i) The Position of On-Board Unit (OBU): The OBU is prepared on a vehicle for between vehicles communications for the different area or communications between the vehicle and roadside units identified. Radio wire is equipped in an OBU to such an extent that the vehicle communications with various side or the roadside units can be applied.

(ii) The Position of Road Side Unit (RSU): The RSU is distributed along the roads. The principal function of the roadside units is to sidestep the messages among the vehicles and trust authority.

(iii) Major Trust Authority (MTA): The MTA is a server, which is overseen by a specialist co-op or the legislature. The function of a trusted authority is to keep up the administration, to keep the records of every vehicle or to issue the testament for every vehicle.

\section{MOBILITY of BBR (BORDER NODE BASED ROUTING)}

In this area, we display a Border routing protocol for in part associated with the different state. The proposed routing is intended for sending messages from a random node to another node (unicast) or from one node to every single other node (broadcast). The general plan objectives are to improve the broadcast conduct for low node thickness and high range mobility. VANET is the advanced technology of structure a robust Ad-hoc network between mobile vehicles, wide side, besides, between mobile vehicles and roadsides units and the routing protocols in VANET areas:

In [4], the routing in Mobile Ad-hoc Networks was described. Routing in mobile Ad-hoc networks is quite a challenging task since the nodes move arbitrarily, have limited power back, topology changes and lack of other resources. In other routing protocols, there is part of routing overhead because of the flooding of the messages. In this paper area following and on request protocol was utilized. There are numerous strategies for finding the portable nodes like Radiolocation procedures, Global Positioning System (GPS), the Bat framework, the Cricket Compass framework and RADAR. This work has made the blend of the area following an instrument that has been designed in the genuine MANET testbed with geo throwing ability. Every portable node (MN) has run the area following system and put away the situating table at the nodes itself in a record. The information was in succession and began with the MAC address, IP address and the separation to each neighbor from MN.

In [5], a protocol named GeoTORA was proposed, as it was gotten from the Temporarily Ordered Routing Algorithm (TORA) (unicast) routing protocol. Flooding is additionally joined in GeoTORA, however, it is restricted to nodes inside a little area. This integration of TORA and flooding can fundamentally diminish the overhead of geocast delivery while keeping up sensibly high exactness. TORA is one of a group of connection inversion algorithms for routing in ad hoc networks. For every conceivable goal in the ad hoc network, TORA keeps up a goal arranged directed acyclic graph (DAG).

In [6], a protocol was suggested that expands existing geocast protocols by supporting a packet delivery framework with adequate flooding. In basic flooding, there are repetitive retransmissions of geocast messages, expanding network traffic, possibly bringing about broadcast storms. The paper proposed an answer for this issue as takes after. Instead of having, all nodes partake in the packet transmission, just the nodes that fulfil following two conditions: those that have a transmission go that is bigger with respect to the normal transmission run, and those that can cover areas that are yet revealed. In this way, this approach diminishes the number of transmissions. To stop a node tolerating copy packets, a one of a kind succession numbers related with every packet, which is contrasted and already recorded (source, grouping) sets.

In [7] Vehicular delay-tolerant network (VDTN) engineering was acquainted with manage availability limitations. VDTN expect offbeat, package situated communication, and a store-convey and-forward routing paradigm. A routing protocol for VDTNs should make the best utilization of the tight assets accessible in network nodes to make a multijump way that exists after some time. A VDTN routing protocol, called GeoSpray, was proposed, which takes routing choices based on geographical area information, and consolidates a hybrid approach between different duplicate and single-duplicate plans. In the first place, it begins with a numerous duplicate plan, spreading a set number of package duplicates, keeping in mind the end goal to abuse elective ways.

In [8] GeoCross, a basic, yet novel, occasion driven geographic routing protocol is suggested that expels crossinterfaces progressively to abstain from routing circles in urban Vehicular Ad Hoc Networks (VANETs). GeoCross 


\title{
International Advanced Research Journal in Science, Engineering and Technology
}

\author{
Vol. 5, Issue 9, September 2018
}

misuses the regular planar component of urban maps without falling back on bulky planarization. Its component of dynamic circle identification makes GeoCross reasonable for profoundly versatile VANET. We have likewise demonstrated that storing (GeoCross + Cache) gives a similar high packet delivery ratio yet utilizes fewer bounces.

In [9] distinctive VANET applications are considered to make the routing protocol. The solace application drives the dangers of new amusements for vehicular ad-hoc networks (VANETs). The satisfaction application generally keeps the delay-tolerant office; that is, messages started from a particular vehicle at time $t$ can be conveyed through VANETs to a few vehicles inside a given obliged delay time $\lambda$. In creator new mobicast, the protocol is examined to help satisfaction applications for an expressway situation in vehicular ad-hoc networks (VANETs). All vehicles situated in a geographic zone at time $t$, the mobicast routing is to scatter the information message started from a particular vehicle to all vehicles, which have ever shown up in the zone at time $t$.

In [10] two extraordinary, supposed, Geocast protocols for VANETs are outlined and assessed. One protocol is intended for quick communication over an extensive area. The motivation behind the other protocol is to give a routing administration to a future dependable transport protocol (empowering Internet applications). The execution of the protocols is assessed utilizing sensible network and traffic models. Distributed Robust Geocast (DRG), is a Geocast protocol that is totally distributed, without control overhead and state data.

\section{A. Improved Edge- Border Node Based Routing (EBBR) Protocol in VANETS}

In our proposed Edge count, measurement for border nodes, which take after by the framework scope detecting separation in BBR protocol, can endure network segment because of low node thickness and high node mobility. The execution of pestilence routing and BBR are assessed with a Geographic and Traffic Information (GTI) based mobility show that catches run of the mill highway conditions. The simulation comes about to demonstrate that under country network conditions, a restricted flooding protocol, for example, BBR performs well and offers the advantage of not depending on an area benefit required by different protocols proposed for VANETs.

We propose new algorithms to consolidate the probabilistic and separation plots keeping in mind the end goal to abuse the advantages of both methodologies. In this paper, the probabilistic plan is called basic probabilistic plan or mode 1 and we propose new modes to enhance this one Density Aware Probabilistic Flooding (mode 2) keeping in mind the end goal to dodge issues with the variety of topology in the network, this change utilizes some data about the area topology. Thus, a node that tunes in to the medium can know its neighbors, and after that the local thickness. A host will rebroadcast flooding messages with the likelihood $p=$ fmode2(n):

$$
\mathrm{F}_{\text {mode }}=\mathrm{K} / \mathrm{N}
$$

where $\mathrm{k}$ is a productivity parameter to accomplish the reachability of the broadcast. The fmode 2 has been picked in light of the fact that, naturally, the ideal likelihood of broadcast is the converse of the local thickness. Moreover, we have watched the accuracy of this presumption.

B. Border retransmission based probabilistic flooding

The past models have the disadvantage to be locally uniform. In reality, every node of a given area gets a broadcast and decides the likelihood as indicated by a steady or from the local thickness. This is like an additional area scope plot [5], yet that plan is position based. We calculate that the separation between two nodes with full duplex communication can be assessed by contrasting their neighbor records and Zsrc(Source area for zone an) and Zdest(destination area for zone an) as far the crossing point between Zsrc(Source area for zone b) and Zdest(destination area for zone b) and in c zone all border node from source to goal beneath in 3 conditions :

$$
\begin{aligned}
& Z \mathrm{Za}=\mathrm{Zsrc} \cap \mathrm{Zdest}: \text { the communication area secured just by src, } \\
& \mathrm{Zb}=\mathrm{Zsrc} \cap \mathrm{Zdest}: \text { the communication area secured just by dest, } \\
& \mathrm{Zc}=\mathrm{Zsrc} \cap \mathrm{Zdest}: \text { the communication area secured by both src and dest, }
\end{aligned}
$$

The nodes can't assess the zones $\mathrm{Za}, \mathrm{Zb}$ and $\mathrm{Zc}$ without situating offices in condition 2.2, 2.3 and 2.4. Be that as it may, these areas can be described by the number of mobiles inside every one of them: the neighbors of src ( $\mathrm{Na}$, number of nodes inside the $\mathrm{Za}$ ) the neighbors of dest $(\mathrm{Nb}$, number of nodes inside the $\mathrm{Zb}$ ) and the neighbors of src and dest $(\mathrm{Nc}$, number of nodes inside the $\mathrm{Zc}$ ). This approach gives a decent guess in the uniform case for the separation between the src and dest. We characterize the ratio $\mu$ by:

$$
\mu=\mathrm{Nb} /(\mathrm{Nb}+\mathrm{Nc})
$$

ast adds the identities 1 of all its neighbors in the message. 


\section{International Advanced Research Journal in Science, Engineering and Technology}

Vol. 5, Issue 9, September 2018

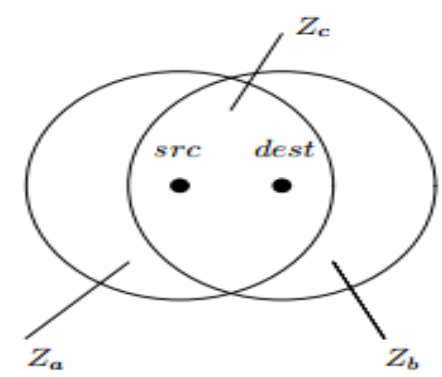

Fig. 2 Connection of the radio areas of nodes src and dest position.

C. Density-based border node retransmission based probabilistic flooding neighbor elimination

In a few conditions, a gathering can be assessed by the maximal estimation of the ratio (Zsrc $\operatorname{lest}$ )/Zsrc which relate to the situation when the separation amongst src and dest is equivalent to the transmission span.

Numerically, $M=13+\sqrt{ } 32 \pi \sim=0$, and a few hubs won't be reached. In the most pessimistic scenarios, a segment of the network can happen regardless of whether the missed nodes are reachable. An answer introduced in [6] depends on a neighbor end conspire every vehicle checks if every one of the neighbors has gotten the communicated message. For the modes 3 et 4, every node which forward the communication message incorporates the rundown of its neighbors. The collector can distinguish which nodes have been secured by checking the neighbor rundown of the transmitter, contrasting, and its own neighbor list.

\section{PROPOSED ALGORITHM}

In our proposed algorithm, each vehicle has own broadcast table VBT, where identifier able to find for each broadcast already received or not for the same id.

1. protocol BBICR()

2. \{

3. IF messages can receive for the initial iteration

4. find the Broadcast ID from the initial message

5. THEN

6. Create initial execution BTbid in the Broadcast Table for roadside distance.

7. Generate a list Lbid with all the IDs in the neighbor road distance table.

8. Fetch route with avg density of vehicle in minimum distance grid or area;

9. Set parameter state $=1$

10. While (overlap $==1$ )

11. Calculate grid sensing ratio GSR;

12. Vehicle overlap optimal angle with 90 (degree towards left or right)

13. if (network is constant)

14. overlap $=\min 3$ vehicles at once;

15. End

16. End

17. calculate GSR;

18. while(GSR >=predefined threshold vehicle count)

19. calculate priority;

20. if(priority is highest for a void road)

21. state $=0$;

22. send state information to its neighboring Vehicle

23. Else

24. calculate GSR(Grid sensing ratio) from vehicle to the next upcoming vehicle ;

25. End if

26. FOR EACH

27. Vehicle id included the same message

28. DO

29. IF id included the same grid in Lbid

30. remove id from Lbid

31. END IF

32. END FOR

33. \} 


\section{International Advanced Research Journal in Science, Engineering and Technology}

Vol. 5, Issue 9, September 2018

\section{SIMULATION RESULTS}

In simulation results, VANET network has been implemented using MATLAB. The performance of the proposed approach evaluated and compared with an earlier work, including intelligence into a VANET and finding the unique grid to improve safety, makes use of V2V and V2R communication.

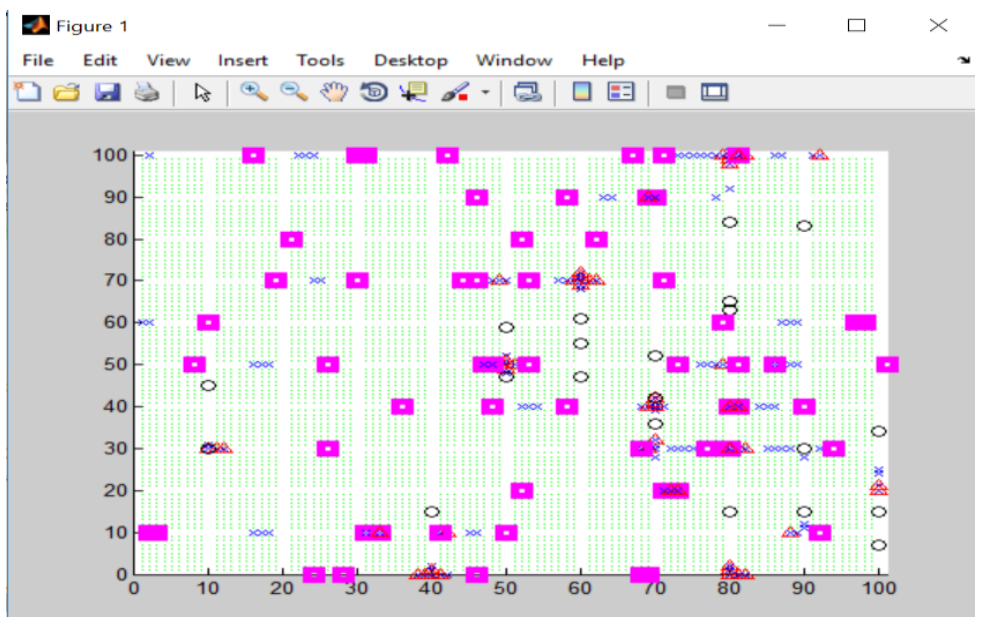

Fig. 3 VANET network simulation with congestion and collision problem.

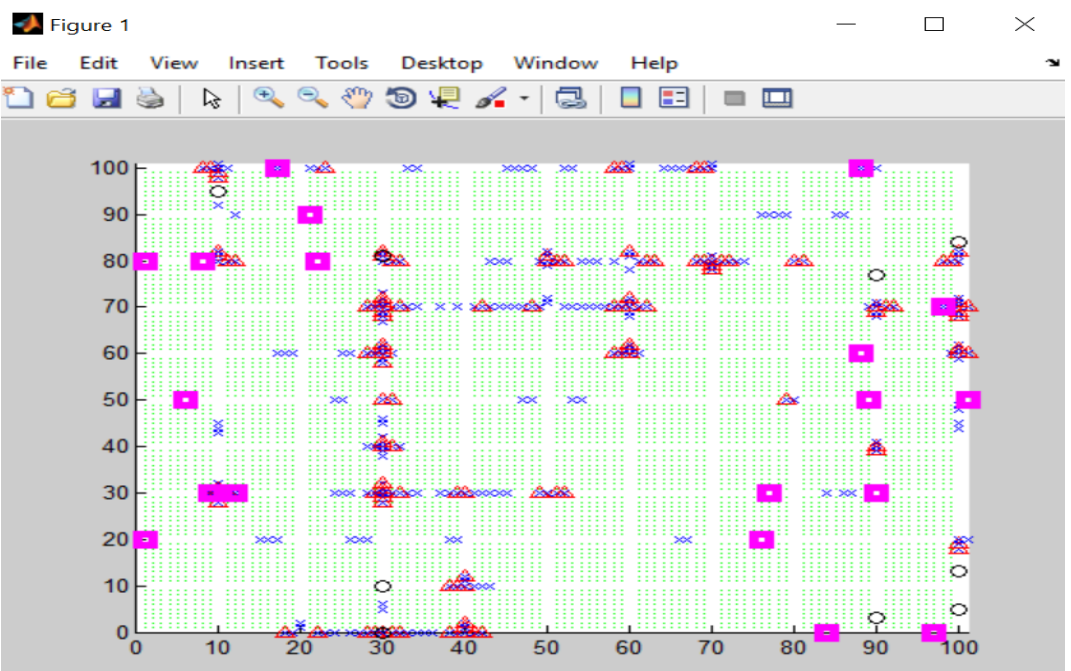

Fig. 4 Proposed VANET network simulation without congestion and collision using the hybrid approach

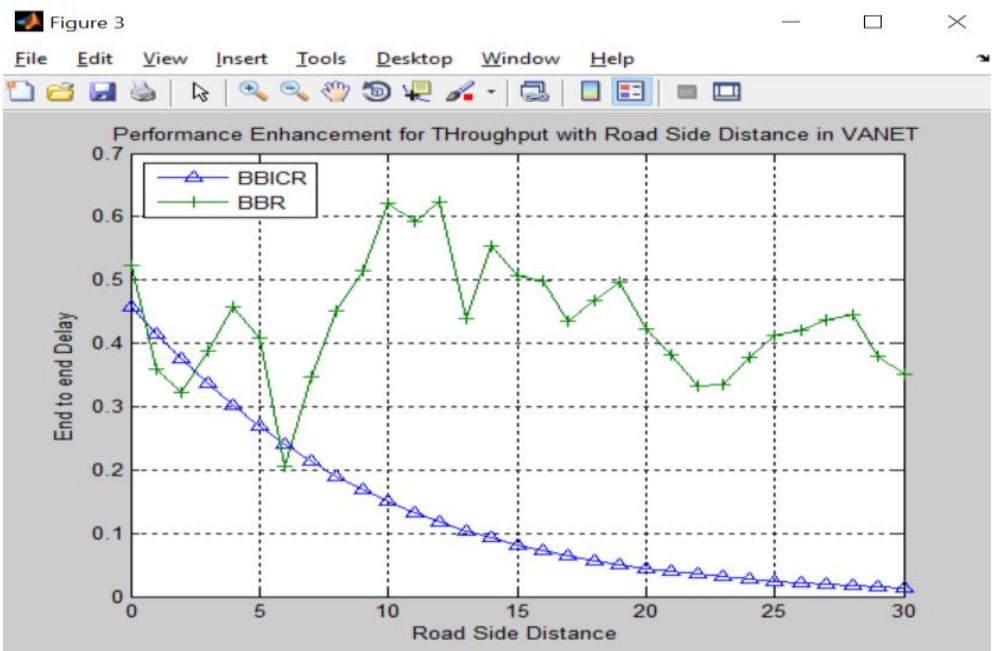

Fig. 5 End to End delay with roadside distance for a proposed hybrid protocol. 


\section{International Advanced Research Journal in Science, Engineering and Technology}

Vol. 5, Issue 9, September 2018

Table 1 Performance Table For The Roadside Distance Of The Proposed Approach With The Existing Algorithm

\begin{tabular}{|l|l|l|}
\hline \multirow{2}{*}{ Road Side Distance } & \multicolumn{2}{|l|}{ TECHNIQUE NAME(End to End Delay) } \\
\cline { 2 - 3 } & BBICR & BBR \\
\hline 5 & 0.28 & 0.41 \\
\hline 10 & 0.15 & 0.61 \\
\hline 15 & 0.089 & 0.50 \\
\hline 20 & 0.05 & 0.42 \\
\hline 25 & 0.02 & 0.4 \\
\hline 30 & 0.01 & 0.35 \\
\hline
\end{tabular}

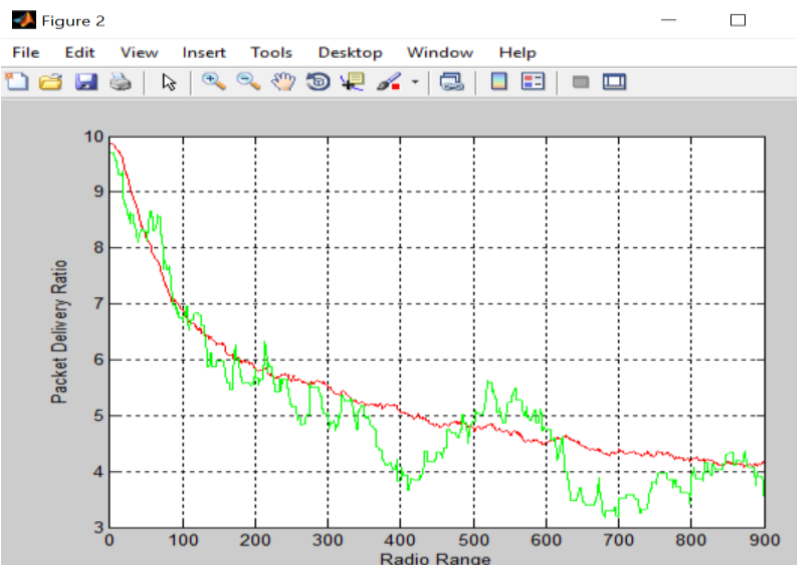

Fig. 6 VANET for packet delivery ratio at different radio range at the minimum distance

Above Fig. 6 Improve traffic safety and comfort of driving at different route with the fixed threshold and Minimize DELAY, traffic intensity, locating vehicles from one side road for proposed protocol.

Table 2 Performance Table For Radio Range Of The Proposed Approach With The Existing Algorithm

\begin{tabular}{|l|l|l|}
\hline \multirow{2}{*}{ RADIO RANGE } & \multicolumn{2}{|l|}{ TECHNIQUE NAME(PDR) } \\
\cline { 2 - 3 } & BBICR & BBR \\
\hline 100 & 7.0 & 7.1 \\
\hline 200 & 6.2 & 6.0 \\
\hline 300 & 5.6 & 4.8 \\
\hline 400 & 5.1 & 3.8 \\
\hline 500 & 4.8 & 4.8 \\
\hline 600 & 4.6 & 4.6 \\
\hline 700 & 4.4 & 3.1 \\
\hline 800 & 4.1 & 3.7 \\
\hline 900 & 4.08 & 3.91 \\
\hline
\end{tabular}

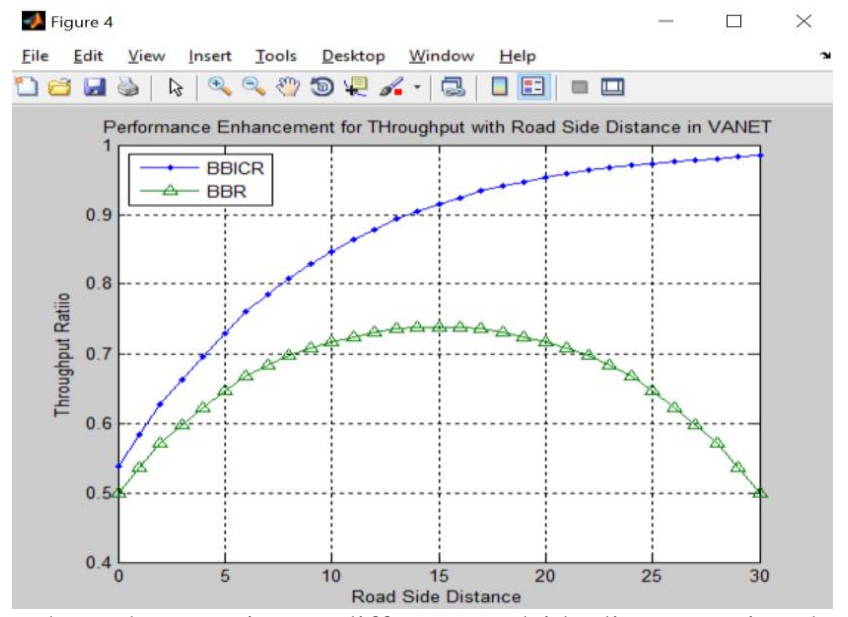

Fig. 7 VANET for Throughput Ratio at a different roadside distance using the hybrid approach 


\section{International Advanced Research Journal in Science, Engineering and Technology}

Vol. 5, Issue 9, September 2018

Table 3 Performance Table for Roadside Distance Based Throughput Ratio of the Proposed Approach with the Existing Algorithm

\begin{tabular}{|l|l|l|}
\hline \multirow{2}{*}{ Road Side Distance } & \multicolumn{2}{|l|}{ TECHNIQUE NAME(Throughput Ratio) } \\
\cline { 2 - 3 } & BBICR & BBR \\
\hline 0 & 0.58 & 0.50 \\
\hline 5 & 0.74 & 0.65 \\
\hline 10 & 0.85 & 0.70 \\
\hline 15 & 0.91 & 0.73 \\
\hline 20 & 0.95 & 0.71 \\
\hline 25 & 0.98 & 0.63 \\
\hline 30 & 0.991 & 0.5 \\
\hline
\end{tabular}

\section{CONCLUSION}

VANET network simulation bunching of neighbors will not rebroadcast the message. In this way, a few nodes will not be reached. In the most pessimistic scenarios, a segment of the network can happen regardless of whether the missed nodes are reachable. An answer is based on a neighbor disposal plot: every node checks if every one of the neighbors has gotten the broadcast message. The packet delivery ratio, throughput and end-to-end delay significantly improved, however, in BBICR, the transmission range of the source is divided into grid segments with radio range. It means that there is almost only one vehicle in a division of network area.

In future, Tensor data can enable an $\mathrm{n}$ way dimensional structure to identify visible or non-visible based vehicular network.

\section{REFERENCES}

[1]. A. Nandan, S. Das, G. Pau, and M. Gerla, "Co-operative downloading in vehicular Adhoc wireless networks," in Proceedings Second Annual IEEE Conference on Wireless On-demand Network Systems and Services (WONS’05), St. Moritz, Switzerland, January 2005 , pp. $32-41$.

[2]. Al-Sultan S, et al. "A comprehensive survey on Vehicular Ad Hoc network", Journal of Network and Computer Applications (2013), http://dx.doi.org/10.1016/j.jnca.2013.02.036i.

[3]. Courtesy/http://adrianlatorre.com/projects/pfc/img/vanet_full.jpg.

[4]. Latiff L, et al. "Location-based Geocasting and Forwarding (LGF) Routing Protocol in Mobile Ad Hoc Network", Proceedings of the Advanced Industrial Conference on Telecommunications/Service Assurance with Partial and Intermittent Resources Conference/eLearning on Telecommunications Workshop, 2005 IEEE.

[5]. Y. Ko and N.H. Vaidya, "GeoTORA: A protocol for geocasting in mobile ad hoc networks", Proc. of the 8th International Conference on Network Protocols, November 2000.

[6]. Hughes L, Maghsoudlou A. "An Efficient Coverage-based Flooding Scheme for Geocasting in Mobile Ad hoc Networks", Proceedings of the 20th International Conference on Advanced Information Networking and Applications (AINA'06), 2006 IEEE.

[7]. V.N.G.J. Soares et al., "GeoSpray: A geographic routing protocol for vehicular delay-tolerant networks", Informat. Fusion (2011), doi:10.1016/j.inffus.2011.11.003.

[8]. Lee K, et al, "GeoCross: A geographic routing protocol in the presence of loops in urban scenarios, Ad Hoc Netw", doi:10.1016/j.adhoc.2009.12.005, 2010.

[9]. Chen Y, et al. "Mobicast Routing Protocol with Carry-and-Forward in Vehicular Ad Hoc Networks", Department of Computer Science and Information Engineering, National Taipei University, Taipei Taiwan.

[10]. MariaKihl, Mihail L. Sichitiu, and Harshvardhan P. Joshi, "Design and Evaluation of two Geocast protocols for Vehicular Ad-hoc Networks", Swedish Governmental Agency for Innovation Systems (Vinnova), February 28, 2008. 